\section{An extension of assimilation theory to illusions of size, area, and direction}

\author{
A. W. PRESSEY* \\ University of Manitoba, Winnipeg, Manitoba, Canada
}

Two postulates, one concerning assimilation and the other concerning attentive fields, are employed to explain the Ponzo, Poggendorff, Wundt, and Hering illusions. Several new configurations are predicted from the theory. In addition, changes in the magnitude or direction of illusion which result from alterations of the basic illusion are explained.

In 1967 , a rudimentary assimilation theory of geometric illusions was proposed (Pressey, 1967). The term, assimilation, expresses the idea that, in a group of en tities, the extremes take on the properties (or become more like) the average of the entities. Assimilation has had a long history of use as an explanatory construct in psychology and has been labeled by other names, including "the central tendency effect," "regression to the mean," and "leveling."

Assimilation is readily demonstrable in a situation in which judgments of various magnitudes are made repeatedly. If, for example, an individual makes several judgments of intervals of $5,10,15$, and $20 \mathrm{sec}$, he will tend to judge the $5 \cdot \mathrm{sec}$ interval as longer than it really is, and the $20-\sec$ interval will be judged as shorter than it is objectively. This principle can be stated as the major postulate of assimilation theory.

Postulate 1. Whenever judgments are made of a series of magnitudes, the smaller magnitudes in that series will be overestimated and the larger magnitudes will be underestimated.

An example of the manner in which

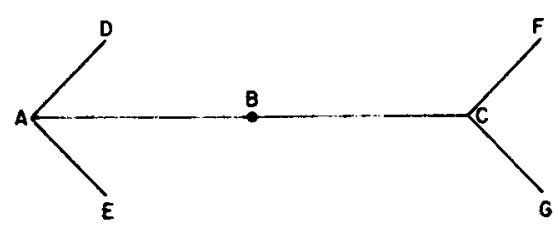

Fig. 1.

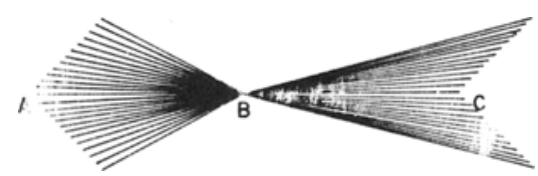

Fig. 2.

*Present address: Department of Psychology, University of Manitoba, Winnipeg 19, Canada. assimilation theory is applied to illusions is provided by the modified Müller-Lyer target shown in Fig. 1. A standard line, bound by an arrowhead at one end and an arrowfeather at another, is bisected by a dot. The objective midpoint appears displaced toward the arrowhead. Assimilation theory states that judgments of the length $A B$ are embedded in a context of judgments of other lengths defined by the obliques $\mathrm{AD}$ and $\mathrm{AE}$. This idea is clarified in Fig. 2. Here $A B$ is the longest line in the series and will be underestimated, whereas $B C$ is the shortest line in its context and will be overestimated. Thus, to bisect the line subjectively, the $\mathrm{O}$ must place his midpoint somewhat toward the arrowfeather side of the POE.

An important modification of the Müller-Lyer illusion is provided in Fig. 3 in which the distance $A B$ appears shorter than the distance $\mathrm{BC}$. This illusion is important because it leads to an extension of assimilation theory. As it stands, assimilation theory is ambiguous about what should occur in Fig. 3 because, logically, the context can be formed by either $A D$ and $A E$ or by $A F$ and $A G$. However, since $\mathrm{AB}$ appears shorter than $B C$, it must be $A D$ and $A E$ (i.e., the arrowheads) which form the effective ' context. And the reason for this might be due to aitentional factors. Consider a nonillusory target such as Fig. 4 in which the $\mathrm{O}$ compares $\mathrm{AB}$ and $\mathrm{BC}$. In his comparison he will probably focus his attention primarily in the region bounded by the points $A$ and $C$. A rough approximation of his attentive field might be as shown by the dotted line. Now, if Fig. 4 is superimposed on Fig. 3, it will be seen that the arrowheads fall within the attentive field and the arrowfeathers fall outside the attentive field. As such, it is highly likely that it is the arrowheads which form the context within which the line $A B$ is embedded.

On the basis of Fig. 4, a second major postulate of assimilation theory is proposed.

Postulate 2. Other things being equal, a

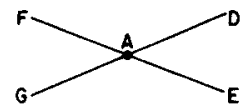

Fig. 3.

context which falls within the attentive field will be more effective than a context outside that field.

There is one major problem with Postulate 2, that is, to specify the boundaries of the attentive field. This is difficult to accomplish precisely, but two methods which might give rough approximations do suggest themselves. The first is an empirical one and the second a logical one. Empirically, eye movements might be measured under the assumption that what one looks at is that to which he is attending. The second method is to analyze logically what response is required of the $\mathrm{O}$ and assume that his attention will be focused primarily on the elements that are to be judged. This procedure of approximating the attentive field has been adopted here. Two points, corresponding to the most distant edges of the elements

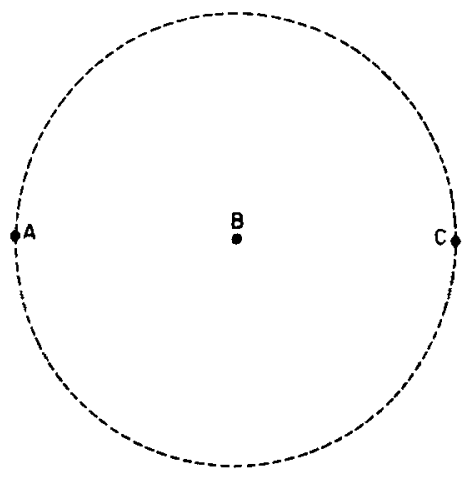

Fig. 4.

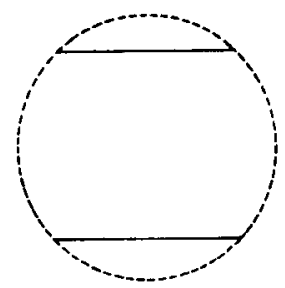

Fig. 5. 


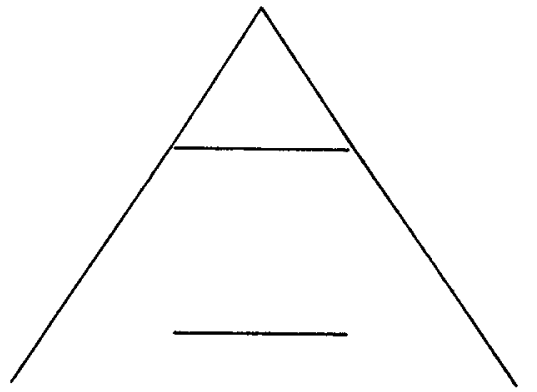

Fig. 6.

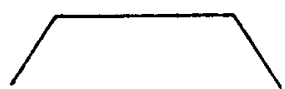

Fig. 7.

to be judged, are joined; this line defines the diameter of a circle. ${ }^{1}$ Elements within that circle are said to be within the attentive field. One example of this method of defining the attentive field has already been given in Fig. 4. A second example is given in Fig. 5 and, if it is granted that the attentive field is roughly as defined, then an explanation of the Ponzo illusion readily follows.

\section{THE PONZO ILLUSION}

The Ponzo illusion is illustrated in Fig. 6. Two horizontal lines of equal length are embedded, one above the other, within an acute angle. The line nearer the apex appears longer than the line below it. An explanation on the basis of assimilation theory follows from a consideration of the attentive field. If Fig. 6 is superimposed on Fig. 5 , it will be seen that certain parts of the obliques fall within the attentive field and the remainder fall outside that field. The contours that fall within the attentive field, and hence provide the most effective context, are shown in Fig. 7. But Fig. 7 is nothing more than an incomplete arrowfeather form of the Müller-Lyer

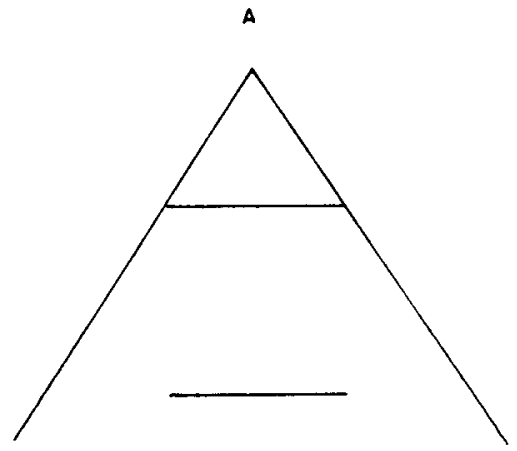

illusion with the same explanation being given (Pressey, 1967). That is, the standard horizontal line assimilates with longer horizontal lines which the arrowfeathers define, with the result that the standard line appears elongated. The bottom horizontal line, on the other hand, does not have effective contours in the attentive field and, as a result, it is judged veridically. Therefore, when the top and bottom lines are compared, the former appears longer.

The targets in Fig. 8 are particularly important because perspective theory, which is the most popular explanation of the Ponzo illusion, and assimilation theory make different predictions about which target should yield the larger illusion.

Perspective theory attributes the distortion exhibited in the Ponzo illusion to the operation of depth-processing mechanisms. That is, the two oblique arms are viewed as two-dimensional perspective cues which yield an appearance of depth. Thus, Gregory (1963) has argued that "The parts of the figures corresponding to distant objects are expanded and the parts corresponding to nearer objects are reduced. Thus .... in the Ponzo figure the upper horizontal line would be farther away and it also is expanded in the flat illusion figure [p. 679]."

It appears that perspective thcory would predict that the target with the "full" acute angle should yield a stronger illusion than the one with an incomplete acute angle. This is because the full angle appears to be a better perspective cue to depth and thus should be more effective in triggering off the depth-processing mechanisms. Assimilation theory, on the other hand, predicts just the opposite effect. This is because the converging lines directly above the upper horizontal line, although outside the attentive field, still have some effect on the length of the standard line. This can be demonstrated by constructing a pattern as shown in Fig. 9. Here, converging lines are located above the upper standard line. In this case the upper line appears shorter

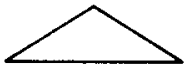

Fig. 9.
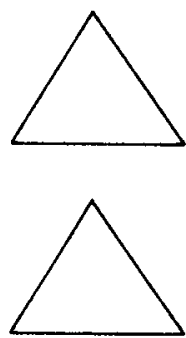

Fig. 10.

than the lower line, though the illusion is not very striking. The shortening effect is due to the assimilation of the standard line to the shorter lines formed by the converging obliques. It is obvious, therefore, that if converging obliques are added above the upper standard line, they will subtract from the effect of the diverging obliques below that line. The net result is that the strongest illusion should be produced by the target shown in Fig. 8B and, as has been verified by many $O s$ in our laboratory, this is indeed the case.

On the $!$ dsis of the above analysis of the Ponzo illusion, other illusory configurations can be constructed. In Fig. 10 two triangles are located one above the other. The baseline of the bottom triangle appears shorter than the baseline of the upper triangle. It is difficult to see how perspective theory would explain this illusion, since there are no obvious cues to depth present. On the other hand,
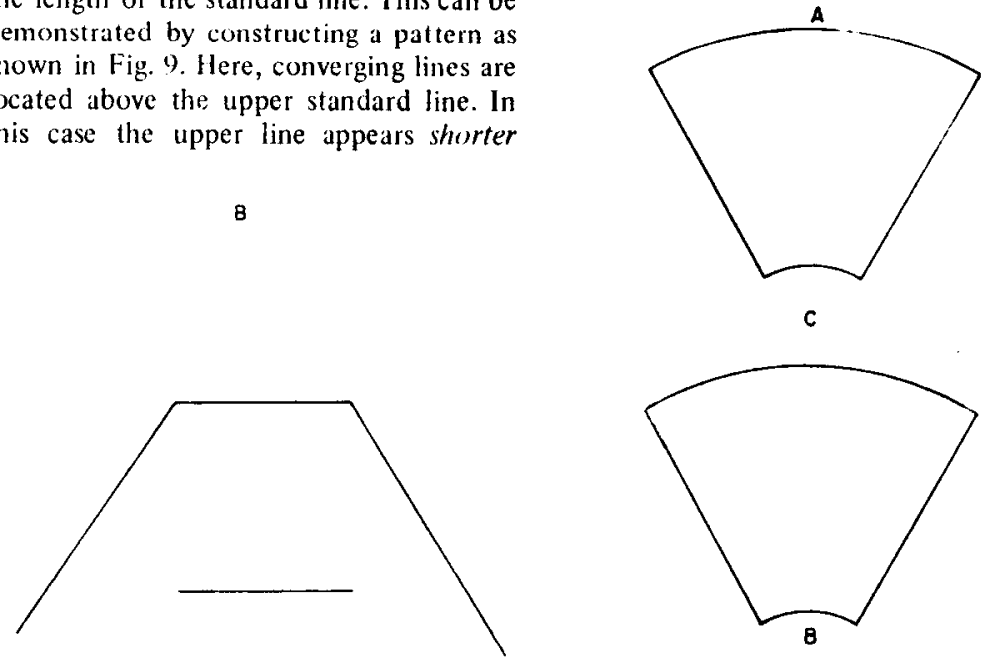

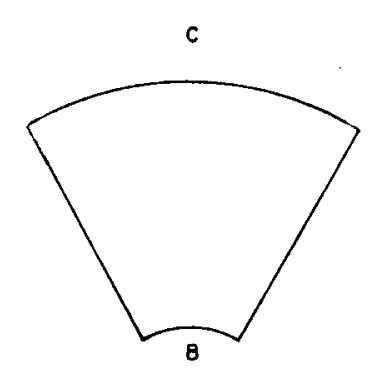

Fig. 11.

Fig. 11.

Fig. 8.

Perception \& Psychophysics, 1971, Vol. 9 (2A) 


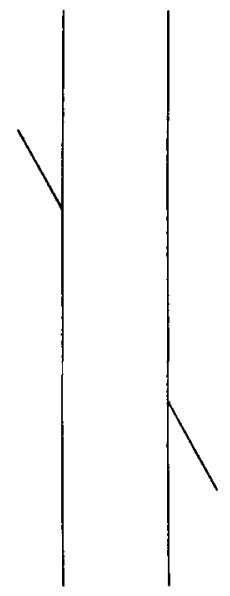

Fig. 12.

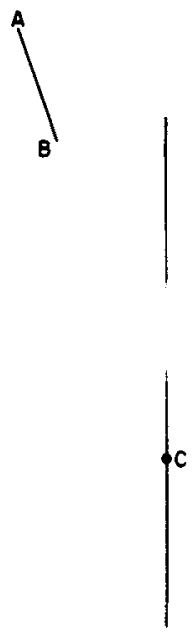

Fig. 13.

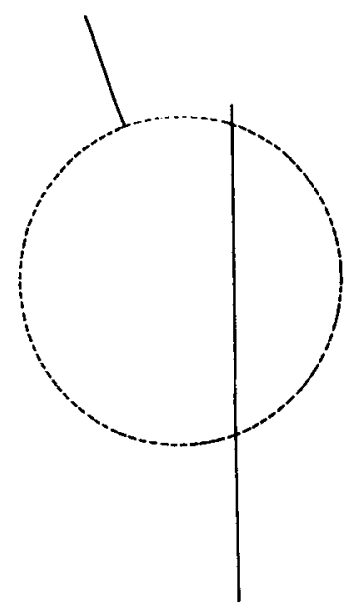

Fig. 14

assimilation theory can explain this illusion on the basis of the attentive field. On the basis of Fig. 5, the ingoing obliques of the lower triangle will be located well within the attentive field and thus should produce a shrinkage of the baseline. The ingoing obliques of the upper triangle, however, are outside the attentive field and thus should produce less shrinkage. Therefore, when the two baselines are compared, the lower one should appear shorter.

\section{WUNDT'S AREA ILLUSION}

The illusion shown in Fig. 10 is similar to the area illusion shown in Fig. 11 (see Tolansky, 1964). The lower figure appears larger than the upper one.

The explanation of this illusion probably lies in attentional factors. The attentive field for Wundt's illusion is different from that of the illusion shown in Fig. 10, because a different response is demanded. If the $\mathrm{O}$ is to judge area, he must scan the entire configuration. Nevertheless, it appears that the center of the field will be attended to the greatest degree. Suppose $O$ scans from $A$ to $B$ in the order $A B A B$; the center of the field $C$ will be represented twice as often as either A or B because the parts processed would be sequentially $A C B C A C B C$. This means that when the area of the lower figure is calculated (by whatever means that is accomplished), the long side will contribute more towards the "calculation." The bottom figure will, therefore, be overestimated. In calculating the upper figure, however, the short base will contribute more (because of differential attention), and hence the area of this figure will be underestimated.

\section{THE POGGENDORFF ILLUSION}

The Poggendorff illusion, illustrated in Fig. 12, is an illusion of direction. The $\mathrm{O}$ is asked to extend an oblique line until it appears to join the farthest vertical line. His projected line deviates systematically from the point of objective continuation.

By introducing a slight modification of the assimilation postulate, an explanation of the Poggendorff illusion is possible. In order to simplify the explanation, a partial illusion which contains no acute angles is employed. In this configuration, shown in Fig. 13, the oblique (AB) appears to point above the $\operatorname{dot}(\mathrm{C})$. This partial illusion has been shown to correlate substantially with the full illusion (Pressey \& Sweeney, 1969).

In order to determine what are the most effective contextual contours, a hypothetical field of attention is drawn as shown in Fig. 14. It is immediately obvious that the dominant contextual contour is that portion of the vertical line which is directly above the point of objective continuation, as shown in Fig. 15. Now, when an $\mathrm{O}$ is asked to subjectively extend the oblique line, he extends a series of lines as shown in Fig. 16. This series of lines consists of lines that are shorter than the

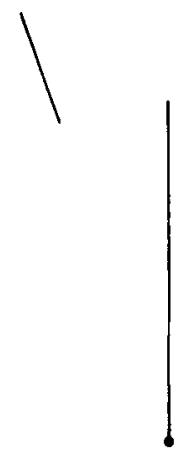

Fig. 15

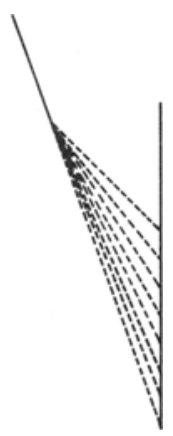

Fig. 16.

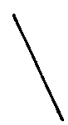

$$
1
$$

Fig. 17.

line of objective continuation. Therefore, his choice of line will assimilate to the mean of the series, which means that he will select a line shorter than the line of objective continuation. But if a shorter line is selected, the oblique line will appear to point above the point of objective continuation, which is precisely what occurs in the Poggendorff illusion.

\section{Reversing the Poggendorff lllusion}

It follows from the above analysis that if one eliminated the portion of the vertical line that forms the effective context (see Fig. 14) and retained only the portion below the point of objective continuation, as shown in Fig. 17, the illusion should reverse. The $\mathrm{O}$ would project a series of lines, but, in this case, he cannot project shorter lines because there is no contour present to form such lines. The only 


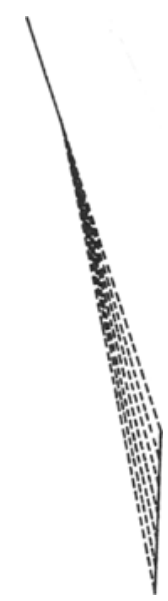

Fig. 18.
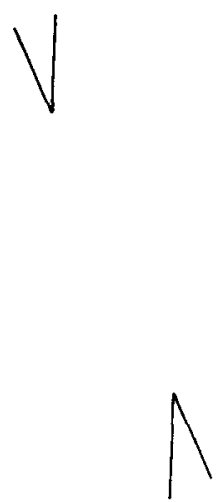

Fig. 19.

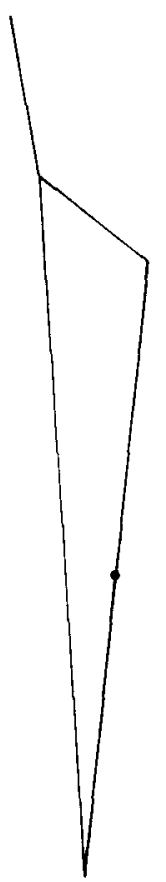

Fig. 20. contour available is one which will form a series of lines that are longer than the line of objective continuation, as shown in Fig. 18. The $\mathrm{O}$ will, therefore, select a longer line, with the result that the oblique line will appear to point below the point of objective continuation. The strength of the reversed illusion, however, should be greatly diminished as compared to the classical illusion, because the context-forming contour is relatively outside the attentive field. There is some empirical evidence to support the prediction of a reversed illusion. Restle (1969) found a reversal in the target shown in Fig. 19 which is, of course, simply a more complex version of Fig. 17.

Another prediction that follows from assimilation theory is that, if Fig. 17 was altered in such a fashion that lines longer than the line of objective continuation were accentuated, the strength of the reversed illusion should increase. Such an accentuation is provided in Fig. 20 in which the oblique line is joined to the bottorn of the vertical contour. As predicted, a striking reversal of the Poggendorff illusion is observed.

\section{Reducing the Poggendorff lllusion}

A marked reduction in the magnitude of the Poggendorff illusion can be produced by altering the orientation of the target (Leibowitz \& Toffey, 1966) and by fixating on the center of the target (Novak, 1966). The assimilation theory can provide an explanation of both of these effects.

If the Poggendorff illusion is oriented so that the oblique line that is to be projected lies on the vertical or the horizontal plane, then the illusion is decreased. The reason for this is that when the $O$ projects a series of lines as shown in Fig. 21, one of these projected lines coincides with the vertical axis. Now, it has been argued by Gibson (1937) and others that the vertical and horizontal directions are "norms" that are highly overlearned. As such, these directional norms would be dominant in any series of directions in which they were embedded. Therefore, in Fig. 21 the projected vertical line would be dominant and not as susceptible to the effects of the remaining lines in the series. Consequently, the individual would tend to choose that dominant line (or one very near to it), with the result that his extension of the short line would be relatively veridical.

Novak (1966) has shown that if an $O$ fixates the center of the target, the Poggendorff illusion decreases. It will be noted in Fig. 22 that this fixation point (X) falls directly on the line that is the objective extension of the oblique. It is possible that because of fixation the objective extension is processed to a higher

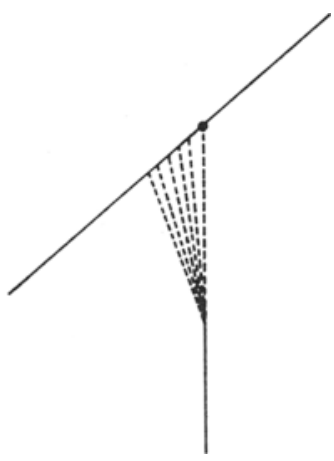

Fig. 21

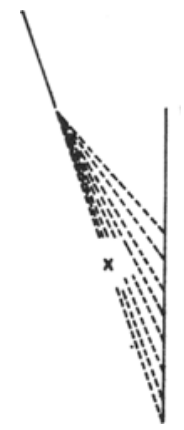

Fig. 22

degree (or is given more weight in the judgme «) than the remaining lines in the series. This would result in a reduction of the illusion.

\section{THE HERING ILLUSION}

In the Hering illusion (Fig. 23) two parallel lines appear bowed if they are located in a context of radiating lines. Many investigators (e.g., Chiang, 1968) have argued that it is the presence of acute angles which causes the illusion. But, as Coren (1970) has shown in Fig. 24, the illusion still occurs when the acute angles formed by the horizontal line are removed.

Postulate 1 is sufficient to provide an explanation of the Hering illusion. The lines $\mathrm{AF}, \mathrm{BF}, \mathrm{CF}, \mathrm{DF}$, and $\mathrm{EF}$ form a series of lines in which $A F$ is the longest and $E F$ is the shortest line. Therefore, AF will be underestimated and $\mathrm{EF}$ will be overestimated. The magnitudes in between these extremes will also be under- and overestimated. Consequently, the entire series of dots will appear bowed.

\section{CONCLUSION}

By a systematic application of an assimilation and an attentive field postulate, the Ponzo, Poggendorff, Wundt, and Hering illusions are explained. The attentive field postulate is a particularly fruitful one, and further research should be 


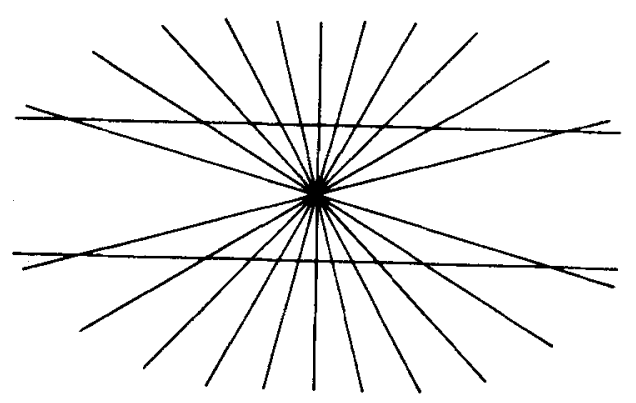

Fig. 23

directed toward an empirical assessment of attentive fields. This could be accomplished by plotting eye movements under the assumption that if a particular portion of the target is viewed more than other parts, it will be more effective in determining the percept.

It should also be noted that the theory presented here does not claim to have isolated all the factors that operate in illusions. It is probable that many factors are involved, with certain ones being more effective than others in different configurations.

\section{REFERENCES}

CHIANG, C. A new theory to explain

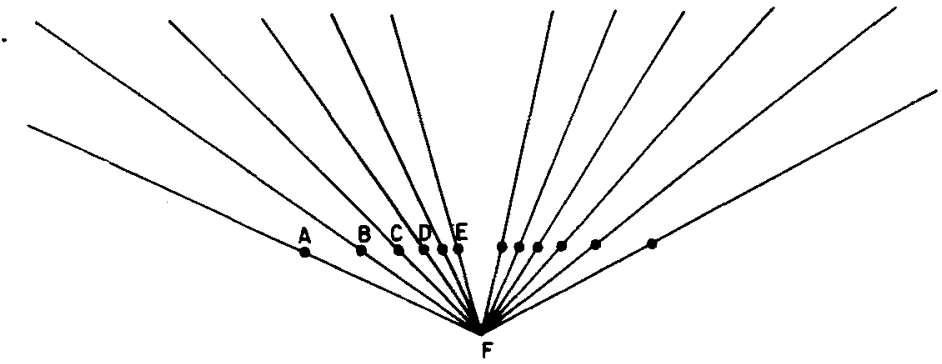

Fig. 24

gcometrical illusions produced by crossing lines. Perception \& Psychophysics, 1968, 3, 174-176.

COREN, S. Lateral inhibition and the Wundt-Hering illusion. Psychonomic Science, $1970,18,341$.

GIBSON, J. Adaptation, after-effect, and contrast in the perception of curved lines: II. Simultaneous contrast and the areal restriction of the after-effect. Journal of Experimental Psychology, 1937, 20, 553-569.

GREGORY, R. L. Distortion of visual space as inappropriate constancy scaling. Nature, 1963, $199,678-680$.

LEIBOWITZ, H., \& TOFFEY, S. The effect of rotation and tilt on the magnitude of the Poggendorff illusion. Vision Research, 1966, 6, 101-103.

NOVAK, S. Effects of free inspection and fixation on the magnitude of the Poggendorff illusion. Perceptual \& Motor Skills, 1966, 23, 663-670.

PRESSEY, A. W. A theory of the Mueller-Lyer illusion. Perceptual \& Motor Skills, 1967, 25, 569-572.

PRESSEY, A. W., \& SWEENEY, O. A variation of the Poggendorff illusion. Perceptual \& Motor Skills, 1969, 28, 883-886.

RESTLE, F. lllusions of bent line. Perception \& Psychophysics, 1969, 5, 273-274.

TOLANSKY, S. Optical illusions. Oxford: Pergamon Press, 1964.

\section{NOTE}

1. The choice of a circle is arbitrary and is used only as an approximation of the attentive field. The shape of the field is probably dependent upon the distribution of the elements in the field.

(Accepted for publication July 6, 1970.) 